\title{
A Grid Based Approach to Analysing Spatial Weighting Matrix Specification
}

\author{
Charles RAHAL ${ }^{\ddagger}$ \\ ${ }^{\ddagger}$ Leverhulme Centre for Demographic Science, University of Oxford, OX1 3UQ \\ E-mail: charles.rahal@sociology.ox.ac.uk
}

8th October, 2017

\begin{abstract}
We outline a grid-based approach to provide further evidence against the misconception that the results of spatial econometric models are sensitive to the exact specification of the exogenously set weighting matrix (otherwise known as the 'biggest myth in spatial econometrics'). Our application estimates three large sets of specifications using an original dataset which contains information on the Prime Central London housing market. We show that while posterior model probabilities may indicate a strong preference for an extremely small number of models, and while the spatial autocorrelation parameter varies substantially, median direct effects remain stable across the entire permissible spatial weighting matrix space. We argue that spatial econometric models should be estimated across this entire space, as opposed to the current convention of merely estimating a cursory number of points for robustness.
\end{abstract}

Keywords: Spatial Econometrics, Real Estate Economics, Model Selection, Data Visualisation

\section{INTRODUCTION}

The most frequently asked question when discussing spatial econometric models regards the sensitivity of the results to the specification of the spatial weighting matrix $(\boldsymbol{W})$. A recent introduction to a special issue of Papers in Regional Science summarizes this succinctly, stating: 'Critics of spatial econometrics almost always in our experience home in on the arbitrary nature of the weights' (Arbia and Fingleton, 2008, p.316). This misconception that spatial regression models are sensitive to $\boldsymbol{W}$ is pervasive and may have arisen from previous research incorrectly interpreting model coefficients as if they were partial derivatives, or from the use of misspecified models (even in cases where the spatial weighting matrix will not theoretically change coefficient point estimates). The perceived issue of sensitivity is highlighted directly within a housing context in an early influential article which claims: 'There does not appear to be any consensus regarding which scheme represents the best realization of the correlation structure appearing in the housing market. This is problematic because all of the results are conditional on the researcher's a priori specification of the spatial structure' (Dubin, 1998, p.88). LeSage and Pace (2014) convincingly conclude that 'the notion that estimates and inferences are sensitive to use of a particular weight matrix as perhaps the biggest myth about spatial regression models' [p.5]. Amongst others, they attack a well cited study (Bell and Bockstael (2000)) which claims that their results are more sensitive to the specification of the spatial weight matrix than to estimation technique. However, for a correctly specified Spatial Error Model (SEM - the type used in that paper), changes in $\boldsymbol{W}$ only change the measures of dispersion: not the point estimates. More generally, methods for specifying $\boldsymbol{W}$ have been discussed extensively, dating back to as early as Kooijman (1976) who proposed choices based on maximizing Moran's coefficient. Much work has since followed aiming to reduce the degree of arbitrariness in the choice of $\boldsymbol{W}$, and LeSage and Parent (2007) develop a Bayesian model averaging procedure (using posterior model probabilities as weights) to incorporate uncertainty. Rather 
than picking (or 'boosting', as per Kostov (2010)) a certain specification, we argue for visualising the 'permissible set' of $\boldsymbol{W}$.

Spatial econometric modeling with real estate sales data provides a particularly interesting challenge due to the need to incorporate the uni-directional nature of time (as opposed to the multidirectional nature of spatial autocorrelation). Within this branch of literature, spatial econometric models have been frequently deployed and often report one or two variants of a spatial model based upon competing weighting matrix specifications. Examples include Can (1990), who compares two weighting specifications ('inverse distance' and 'inverse distance squared'), with an additional two which are limited to distance cut-offs, and Pace et al. (1998) who claim distance based approaches 'subsume two basic paradigms of real estate work' [p.8]. Wilhelmsson (2002) considers four different variants, again within a housing context. Can and Megbolugbe (1997) identify and utilize only (recent) 'comparable sales': properties within an arbitrarily pre-specified distance which sold within an arbitrarily determined 'a priori' time period. A number of related papers explicitly incorporate the temporal distance between sales into the weighting matrix itself (Thanos et al. (2016); Dubé and Legros $(2014,2013)$ ), and this is the approach which we follow within this paper for two of our three sets of models.

The purpose of this paper is twofold. The first objective is to outline a grid based approach which can provide visualisations of sets of model outcomes. This allows a significantly more robust analysis contingent on a large set of inputs which we term the 'permissible set' of specifications, made possible by recent improvements in computer processing power. The second is to provide further evidence against the myth that small changes in the specification of $\boldsymbol{W}$ can dramatically effect modeling outcomes in an application which utilizes a new dataset for 'Prime Central London' (PCL) real estate. Section 2 introduces the dataset and Section 3 discusses the grid based approach. We visualise and analyse the outputs in Section 4 before concluding in Section 5.

\section{DATASET: PRIME CENTRAL LONDON HOUSING MARKET}

We use information on a large number of real estate sales in PCL exclusively from postcode areas SW1, SW3, SW7, W1, W2, W8, W11 and W14. The data is collected from a subscription based archive with contributions from individual realtors which is then manually parsed into a tabular database. It contains a large number of structural characteristics in order to avoid any potential misspecification of the independent variables: a problem which frequently plagues hedonic regressions of this sort. It contains the type of property (terraced, apartment, etc.), number of bedrooms, bathrooms, shower rooms, reception rooms, the condition and whether the property has a garage, (communal) garden, patio or a terrace. Importantly, it contains the 'gross floor area' and the date of sale and a postcode which we subsequently geocode into latitude and longitude using the Googlemaps API. We clean the dataset in accordance with the existing hedonic literature and trim it to contain only sales between 2000-2015, dropping observations in the lower and upper 2.5 percentiles of the sale price. This leaves 4,731 observations. ${ }^{1}$

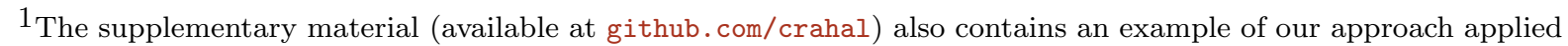
to the infamous Boston housing dataset of Harrison and Rubinfeld (1978), further developing Section 3.3 of LeSage and Pace (2014).
} 
Figure 1: Weighting Matrix Sparsity

(a) Sparsity of $W_{A}^{\alpha, \delta}$ and $W_{B}^{\alpha, \delta}$ Specifications

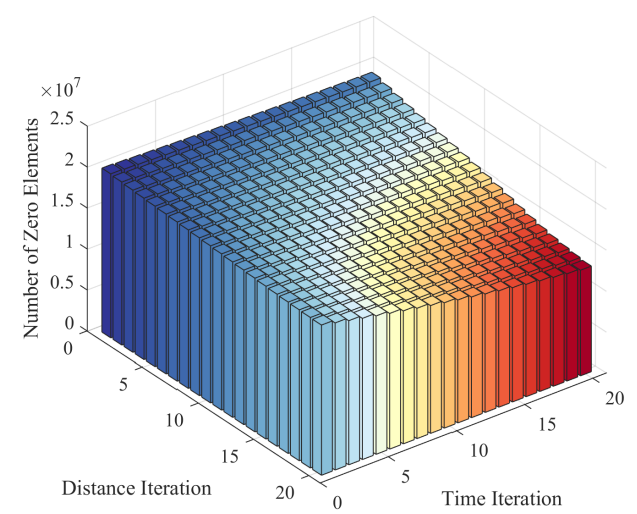

(b) Sparsity of $W_{C}^{\alpha, \delta}$ Specifications

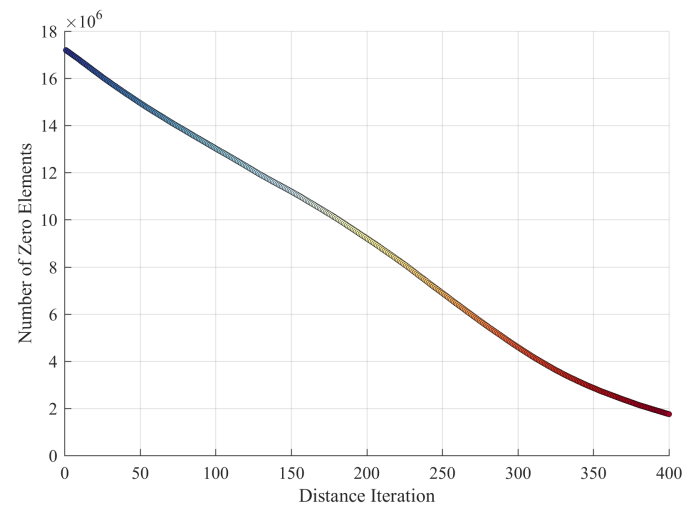

\section{METHODOLOGY}

As the simplest exposition of our grid based approach, we utilize the popular Spatial Autoregression (SAR) model which takes the form:

$$
y=\theta \iota_{n}+\lambda \boldsymbol{W} y+\boldsymbol{X} \beta+\varepsilon
$$

with $\varepsilon \sim N\left(0, \sigma^{2} \boldsymbol{I}_{n}\right)$. In this deliberately simple setup, $y$ is an $N \times 1$ vector of sales prices and $\boldsymbol{X}$ is an $N \times k$ matrix of observations on the explanatory variables (as per Section 2). $\beta$ is a $k \times 1$ vector of coefficients associated with $X$, and $\iota_{n}$ an $N \times 1$ vector of constants. The $N$ disturbances $(\varepsilon)$ are distributed normally with constant variance $\left(\sigma^{2}\right)$ and zero covariance across observations, and $\lambda$ represents a scalar spatial dependence parametre (typically in the interval $[0,1]$ ). While we focus on PCL house prices within a SAR model, this approach applies equally to any spatial econometric approach where $\boldsymbol{W}$ is exogenously specified. We augment $\boldsymbol{X}$ with quarterly dummies in order to account for the upward trend in the PCL market in our sample period.

We examine three sets of matrices which expand the ideology of LeSage and Pace (2014), with the first two incorporating a temporal dimension. We first construct distance $(\mathbf{D})$ and time $(\mathbf{T})$ matrices:

$$
\boldsymbol{D}=\left[\begin{array}{cccc}
0 & d_{1,2} & \ldots & d_{1, j} \\
d_{1,2} & 0 & \ldots & d_{2, j} \\
\vdots & \ldots & \ddots & \vdots \\
d_{1, j} & d_{2, j} & \ldots & 0
\end{array}\right] \quad \boldsymbol{T}=\left[\begin{array}{cccc}
0 & t_{1,2} & \ldots & t_{1, j} \\
-t_{1,2} & 0 & \ldots & t_{2, j} \\
\vdots & \ldots & \ddots & \vdots \\
-t_{1, j} & -t_{2, j} & \ldots & 0
\end{array}\right]
$$

where $d_{i, j}$ is the Haversine distance (in kilometers) between $i$ and $j$ and $t_{i, j}=$ SaleMonth $_{i}$ SaleMonth $_{j}$ is the temporal distance where SaleMonth refers to a categorical, numerical ordering of the months in which properties were sold over time. By design, $\boldsymbol{D}$ is symmetric, and $\boldsymbol{T}$ is symmetric with signs reversed. The first two sets of matrices $\left(\boldsymbol{W}_{A}^{\alpha, \delta}\right.$ and $\left.\boldsymbol{W}_{B}^{\alpha, \delta}\right)$ exploit the 'Hadamard' 
product style of weighting matrix as per Thanos et al. (2016). For $\boldsymbol{W}_{A}^{\alpha, \delta}$, elements $w_{i, j, A}^{\alpha, \delta}$ are defined as:

$$
w_{i, j, A}^{\alpha, \delta}= \begin{cases}\frac{1}{t_{i, j}^{\alpha}} \times \frac{1}{d_{i, j}^{\alpha}} & \text { if } 0<t_{i, j}<\bar{t} \text { and } d_{i, j}<\bar{d} \text { and } i \neq j \\ 1 \times \frac{1}{d_{i, j}^{\alpha}}, & \text { if } 0=t_{i, j} \text { and } d_{i, j}<\bar{d} \text { and } i \neq j \\ 0, & \text { otherwise }\end{cases}
$$

and the traditional decay parameter (discussed below) is denoted $\alpha$. Critically, $\delta$ denotes a function of $\delta_{m}^{T}$ and $\delta_{m}^{S}$, defined as the fraction of the maximum distance along either the time or space dimension: for $\bar{t}$ and $\bar{d}$ where $\bar{t}=\delta_{m}^{T} \times \max _{i \in\{1, \ldots, N\}, j \in\{1, \ldots, N\}}\left\{t_{i, j}\right\}$ and $\bar{d}=\delta_{m}^{S} \times$ $\max _{i \in\{1, \ldots, N\}, j \in\{1, \ldots, N\}}\left\{d_{i, j}\right\} .^{2,3}$ The second matrix, denoted as $\boldsymbol{W}_{B}^{\alpha, \delta}$, applies inverse distance weights (as opposed to inverse distance and time as per $\boldsymbol{W}_{A}^{\alpha, \delta}$ ) and represents a more traditional spatial weighting specification, merely restricted to observations which have already occurred through the natural passage of time. What is of critical importance to re-emphasize at this point is that if one observation happens before another $\left(t_{i, j}<0\right)$, then the element $w_{i, j, A}^{\alpha, \delta}$ and $w_{i, j, B}^{\alpha, \delta}$ is set to zero, highlighting the uni-directional time dimension under examination:

$$
w_{i, j, B}^{\alpha, \delta}= \begin{cases}\frac{1}{d_{i, j}^{\alpha}} & \text { if } 0 \leq t_{i, j}<\bar{t} \text { and } d_{i, j}<\bar{d} \text { and } i \neq j \\ 0, & \text { otherwise }\end{cases}
$$

In both specifications, observations at period $t_{i, j}=0$ might be thought of as 'under offer' or 'subject to completion' within a real-estate context. For our first two specification sets, we estimate across a grid of size $\Omega \times \Omega$ where each location on the grid contains a structure of estimation results for a specific weighting matrix specification related to a specific cut-off ( $\bar{d}$ and $\bar{t}$ as defined above). In our application, our grid contains estimates based on a range of cut-offs contingent on $\delta_{m}^{S}$ and $\delta_{m}^{T}$ across $\left(\delta_{L}+\frac{m \times\left(\delta_{U}-\delta_{L}\right)}{\Omega}\right)$ for $m=\{1, \ldots, \Omega\}$. In the empirical application which follows, we set the lower and upper bounds respectively as $\delta_{L}=0.1$ and $\delta_{U}=0.5$ and our grid to contain 400 points $(\Omega=20)$. That is, for the first two methods, we vary the space $\left(\delta_{m}^{S}\right)$ and time $\left(\delta_{m}^{T}\right)$ cut-offs simulatenously twenty times each, moving linearly between each minimum $\left(\delta_{L}\right)$ and maximum $\left(\delta_{U}\right)$ cuttoff to create 400 variations in total. The third of the three specification sets (to align with papers such as LeSage and Pace (2014) and Kostov (2010)) can be expressed as $\boldsymbol{W}_{C}^{\alpha, \delta^{S}}-$ where we vary $\delta_{m}^{S}$ evenly across $m=\{1, \ldots, 400\}$ to create 400 versions of $\boldsymbol{W}_{C}^{\alpha, \delta^{S}}$ over increasing inverse distance cut-offs $(\bar{d})$. This represents a more conventional spatial (as opposed to spatio-temporal) weighting matrix where each element is constructed as:

$$
w_{i, j, C}^{\alpha, \delta^{S}}= \begin{cases}\frac{1}{d_{i, j}^{\alpha}} & \text { if } d_{i, j}<\bar{d} \text { and } i \neq j \\ 0, & \text { otherwise }\end{cases}
$$

and allows a bi-directional temporal mechanism with no cut-off based on the date of sale.

\footnotetext{
${ }^{2}$ In our PCL example, these maximum elements for $\mathbf{D}$ and $\mathbf{T}$ correspond to $9.904 \mathrm{~km}$ and 191 months respectively (sixteen years of data across twelve months per year).

${ }^{3}$ Conversely, the $\alpha$ subscript represents the matrix elements determined by a specified decay function.
} 
(a) Log Likelihoods $\left(\boldsymbol{W}_{A}^{\alpha, \delta}\right)$

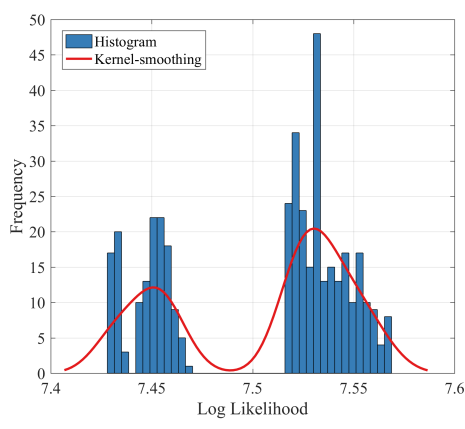

(d) Posterior Probabilities $\left(\boldsymbol{W}_{A}^{\alpha, \delta}\right)$

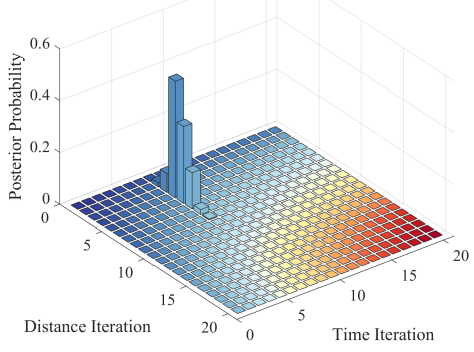

Figure 2: Evaluating Model Fit

(b) Log Likelihoods $\left(\boldsymbol{W}_{B}^{\alpha, \delta}\right)$

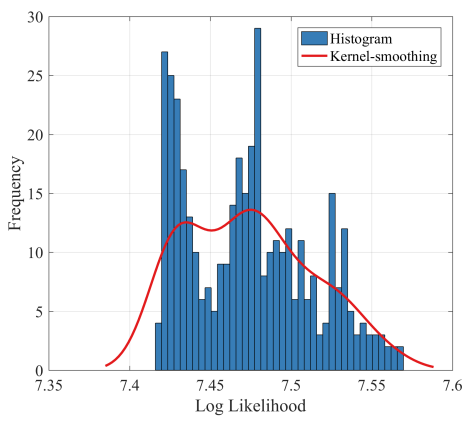

(e) Posterior Probabilities $\left(\boldsymbol{W}_{B}^{\alpha, \delta}\right)$

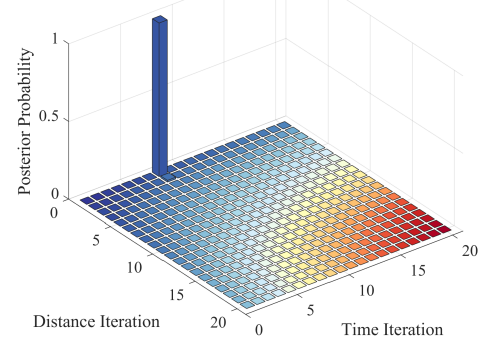

(c) Log Likelihoods $\left(\boldsymbol{W}_{C}^{\alpha, \delta^{S}}\right)$

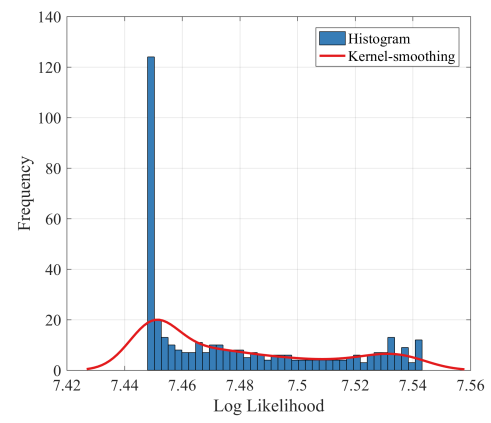

(f) Posterior Probabilities $\left(\boldsymbol{W}_{C}^{\alpha, \delta}\right)$

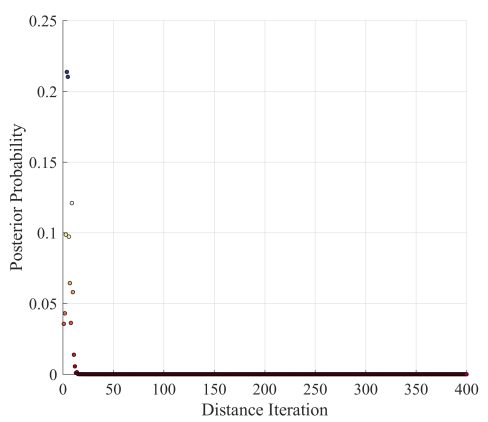

Within our application to PCL data, these three specification sets (visualised as a 'grid' of results across estimations within a SAR model as per Equation 3.1) result in sparsity (the number of non-zero elements in the weighting matrices) structures as shown in Figure $1 .{ }^{4}$ The inclusion of the temporal dimension in the first two specification sets significantly increases the number of zero elements when compared to the spatial specification, and goes some way to addressing the problem of over-connectivity bias. For all three specification sets discussed above, we then re-run the analysis fixing $\bar{t}=\bar{d}=0.5$, but now instead varying $\alpha$ linearly between 0.41 and 4.4 (inclusive) in increments of 0.01 , whereas in the first set of variations across space and time (i.e. ' $\delta$ space'), $\alpha$ is fixed to 1 . These variations represent what might be thought of as the 'permissible space' of all specifications.

\section{ANALYSIS}

We aim to provide further empirical evidence on whether small changes - either through 'boosting' based algorithms or accidental mis-specification - provide substantial differences in effects estimates. We first plot the histograms and kernel density estimates of the distributions of individually maximized log-likelihood values from each weight matrix within each of our three specification sets (Figures 2a-2c) for variations across space and time cut-offs, where each observation represents a single one of the 400 hundred models in each specification set. We then plot a direct (Bayesian) test for model comparison based on (LeSage and Pace, 2009, p. 175-178) in Figures 2e-2f, where

\footnotetext{
$4_{\text {Note that schemes }} \boldsymbol{W}_{A}^{\alpha, \delta}$ and $\boldsymbol{W}_{B}^{\alpha, \delta}$ necessarily result in the same sparsity structures but with different representative weights.
} 
Figure 3: Estimation Results Across $\delta$ Space

(a) $\hat{\lambda}$ Estimates $\left(\boldsymbol{W}_{A}^{\alpha, \delta}\right)$

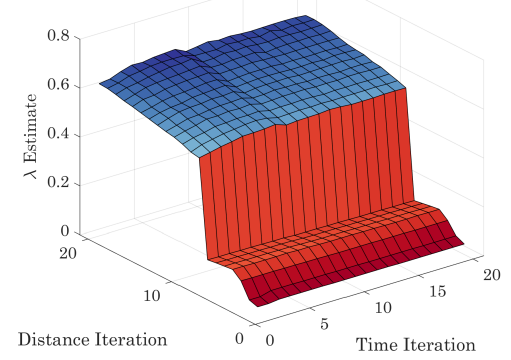

(d) Direct: GFA $\left(\boldsymbol{W}_{A}^{\alpha, \delta}\right)$

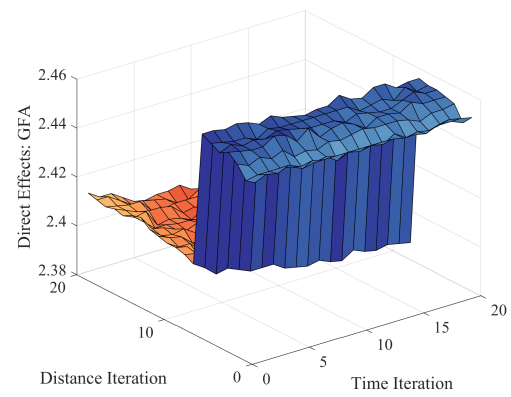

(g) Direct: Detached $\left(\boldsymbol{W}_{A}^{\alpha, \delta}\right)$

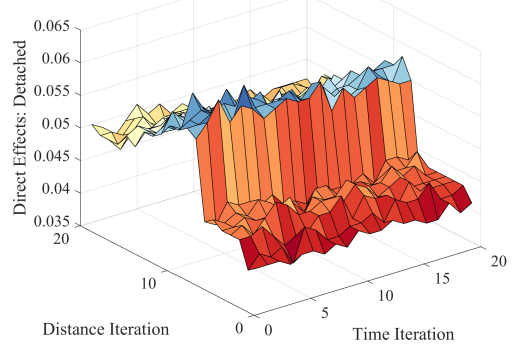

(b) $\hat{\lambda}$ Estimates $\left(\boldsymbol{W}_{B}^{\alpha, \delta}\right)$

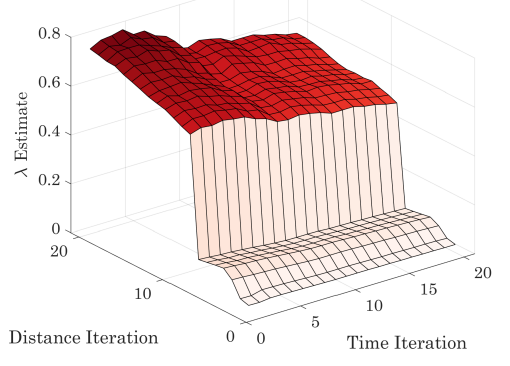

(e) Direct: GFA $\left(\boldsymbol{W}_{B}^{\alpha, \delta}\right)$

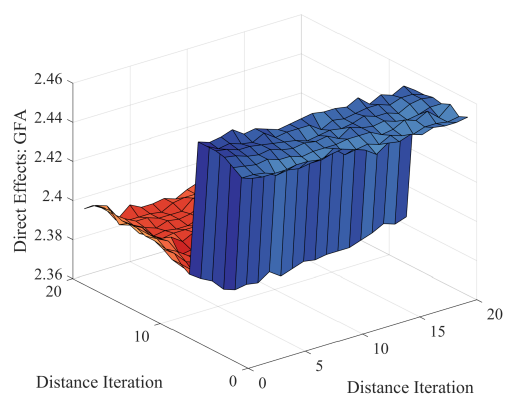

(h) Direct: Detached $\left(\boldsymbol{W}_{B}^{\alpha, \delta}\right)$

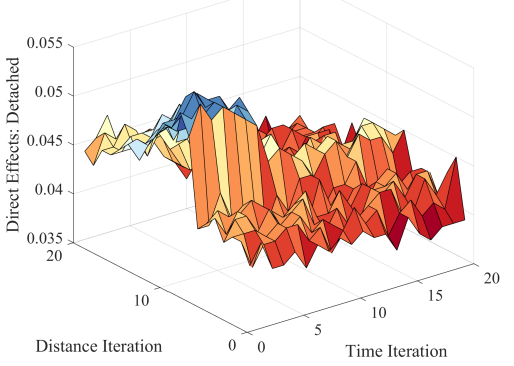

(c) $\hat{\lambda}$ Estimates $\left(\boldsymbol{W}_{C}^{\alpha, \delta^{S}}\right)$

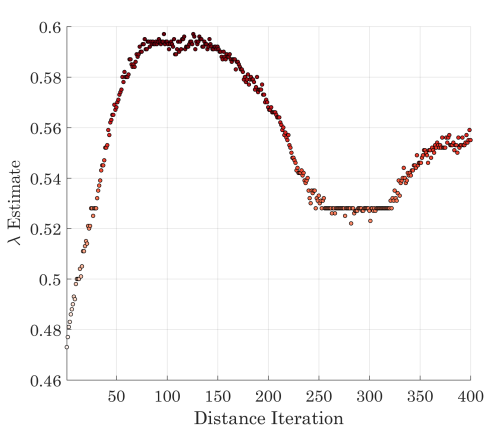

(f) Direct: GFA $\left(\boldsymbol{W}_{C}^{\alpha, \delta^{S}}\right)$

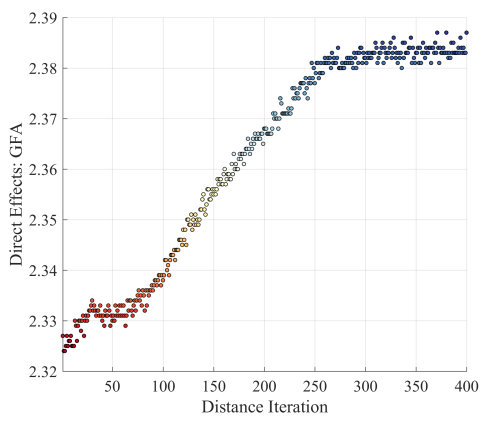

(i) Direct: Detached $\left(\boldsymbol{W}_{C}^{\alpha, \delta^{S}}\right)$

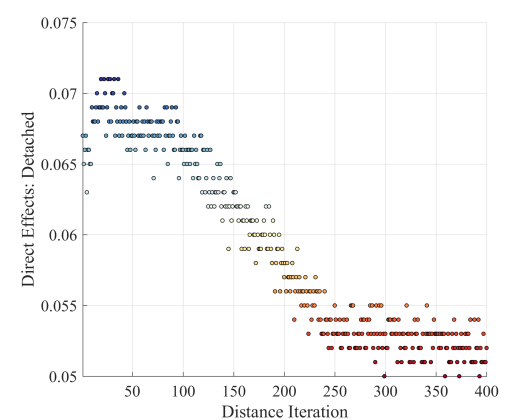

each element on the grid represents a single combination of space $\left(\delta^{S}\right)$ and time $\left(\delta^{T}\right)$ cut-off. ${ }^{5}$ We observe substantial variation in log likelihoods and virtually no posterior probability support for all but a small number of models. ${ }^{6}$ Given such strong support for such a small number of models, we might expect that estimates and inferences might be sensitive to an incorrect choice of weighting matrix specification.

We observe a relatively large variation in estimates for the spatial dependence parameter $(\hat{\lambda})$ in response to changes in the spatial weighting matrix specification (Figures $3 \mathrm{a}$ to $3 \mathrm{c}$ ). This ranges from 0.0397 to 0.4740 for $\boldsymbol{W}_{A}^{\alpha, \delta}, 0.0326$ to 0.7890 for $\boldsymbol{W}_{B}^{\alpha, \delta}$, and 0.4740 to 0.6690 for $\boldsymbol{W}_{C}^{\alpha, \delta^{S}}$. However, as shown in LeSage and Pace (2014), it is the effects estimates which are relevant, not

${ }^{5}$ Each distance iteration is equivalent to approximately $208.5 \mathrm{~m}$ and each time iteration equal to 4.02 months for $\boldsymbol{W}_{A}^{\alpha, \delta}$ and $\boldsymbol{W}_{B}^{\alpha, \delta}$. For $\boldsymbol{W}_{C}^{\alpha, \delta}$, each distance iteration is approximately $10 \mathrm{~m}$.

${ }^{6}$ An unreported figure which compares all 1200 models directly shows that one model (from set $\boldsymbol{W}_{B}^{\alpha, \delta}$ ) obtains a posterior model probability of 0.82 alone. 
Figure 4: Estimation Results Across $\alpha$ space

(a) Posterior Probabilities

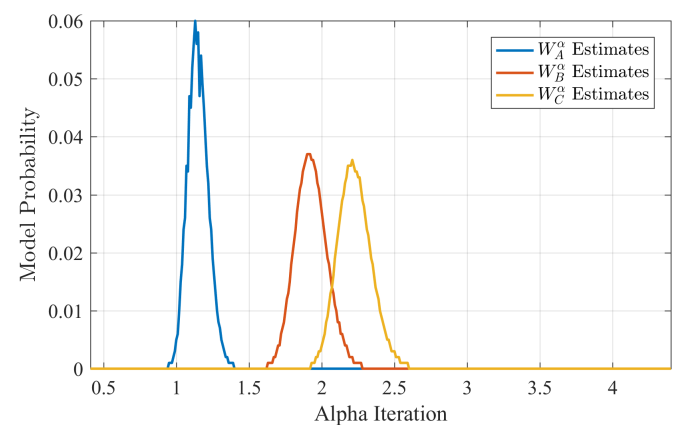

(c) Direct Effects: GFA

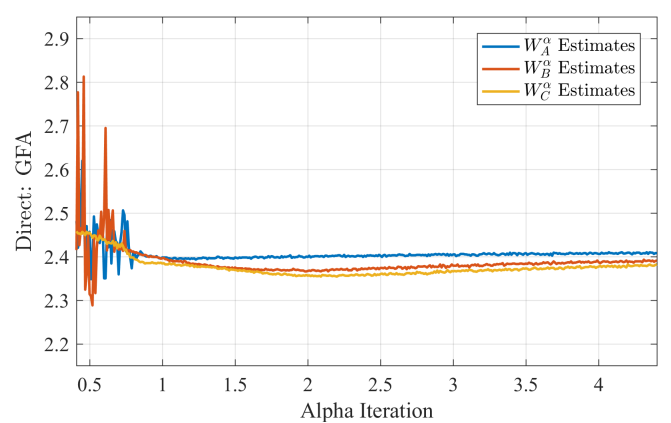

(b) $\hat{\lambda}$ Estimates

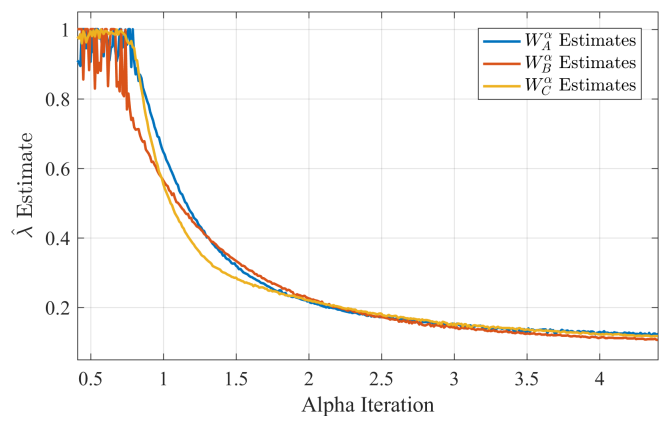

(d) Direct Effects: Detached

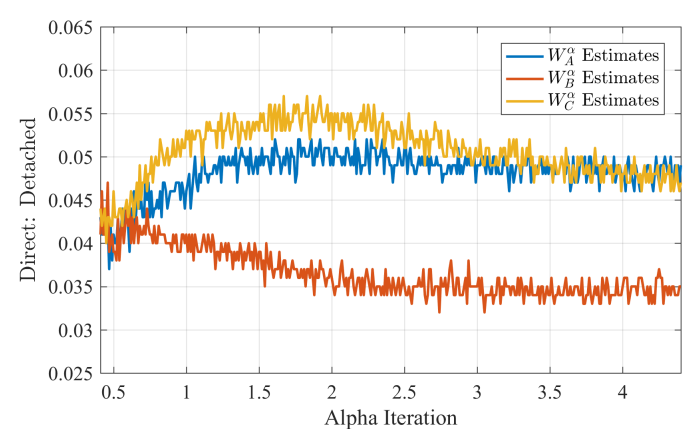

the coefficient estimates: the $\hat{\beta}$ and $\hat{\lambda}$ adjust in response to changes in the weighting specification in an effort to produce consistent effects estimates. In contrast to the $\hat{\lambda}$ estimates in Figures $3 \mathrm{~d}$ to 3i, the direct effects estimates (shown here for the logarithm of Gross Floor Area (GFA) and the 'detached' dummy) across all 1200 specifications are remarkably similar. ${ }^{7}$ Where the results in Kostov (2010) are used to suggest the need to 'fine-tune' weighting matrix specifications, our results (consistent across direct and indirect effects estimates for other variables) show a resilience and robustness across large sets of spatial weighting specifications in our grid of time and space cutoff points. Figure 5 shows the variation across decay parameter with all three of our specification sets now overlaid on top of one another. We again observe the same characteristics in the results: that while only a small number of models have essentially non-zero posterior model probabilities (Figure $4 \mathrm{a}$ ), with $\hat{\lambda}$ varying substantially (Figure $4 \mathrm{~b}$ ), there is relatively little variation in the direct effects estimates (Figures 4c-4d) other than for a small number of models in the $\boldsymbol{W}_{B}^{\alpha, \delta}$ scheme.

\section{CONCLUSION}

We provide further evidence against the 'biggest myth in spatial econometrics' by estimating large sets of different weighting matrix specifications in a new application to modeling PCL house prices. We provide a methodology for visualising large sets of specifications simultaneously, showing that despite the models showing a distinct preference (through log likelihoods and posterior probabilities), direct effects remain largely unchanged. We encourage future researchers to estimate a grid of specifications in order to consider the distribution of parameters across a permissible spatial

\footnotetext{
${ }^{7}$ We use the median of the effects estimates since the effects can have a non-symmetric distribution.
} 
weighting matrix specification space as opposed to estimating a small set of robustness checks in their applications.

\section{ACKNOWLEDGMENTS}

Thanks are due to Anindya Banerjee, Alexandros Rigos, Stephen Hall, William Pouliot, Marco Barassi, John Fender, Rob Elliot and seminar participants at the University of Birmingham. We gratefully acknowledge James Wyatt for providing access to the dataset.

\section{REFERENCES}

Arbia, G. and B. Fingleton (2008). New spatial econometric techniques and applications in regional science. Papers in Regional Science 87(3), 311-317.

Bell, K. P. and N. E. Bockstael (2000). Applying the generalized-moments estimation approach to spatial problems involving microlevel data. The Review of Economics and Statistics 82(1), $72-82$.

Can, A. (1990). The measurement of neighborhood dynamics in urban house prices. Economic Geography 66(3), 254-272.

Can, A. and I. Megbolugbe (1997). Spatial dependence and house price index construction. The Journal of Real Estate Finance and Economics 14(1), 203-222.

Dubé, J. and D. Legros (2013). Dealing with spatial data pooled over time in statistical models. Letters in Spatial and Resource Sciences 6(1), 1-18.

Dubé, J. and D. Legros (2014). Spatial econometrics and the hedonic pricing model: what about the temporal dimension? Journal of Property Research 31 (4), 333-359.

Dubin, R. A. (1998). Spatial autocorrelation: A primer. Journal of Housing Economics 7(4), 304 $-327$.

Harrison, D. and D. L. Rubinfeld (1978). Hedonic housing prices and the demand for clean air. Journal of Environmental Economics and Management 5(1), 81-102.

Kooijman, S. A. L. M. (1976). Some Remarks on the Statistical Analysis of Grids Especially with Respect to Ecology, pp. 113-132. Boston, MA: Springer US.

Kostov, P. (2010). Model boosting for spatial weighting matrix selection in spatial lag models. Environment and Planning B: Planning and Design 37(3), 533-549.

LeSage, J. and R. K. Pace (2009). Introduction to spatial econometrics. Taylor and Francis Boca Raton.

LeSage, J. and R. K. Pace (2014). The biggest myth in spatial econometrics. Econometrics 2(4), $1-33$.

LeSage, J. P. and O. Parent (2007). Bayesian model averaging for spatial econometric models. Geographical Analysis 39(3), 241-267.

Pace, R. K., R. Barry, and C. Sirmans (1998). Spatial statistics and real estate. The Journal of Real Estate Finance and Economics 17(1), 5-13.

Pace, R. K. and O. Gilley (1997). Using the spatial configuration of the data to improve estimation. The Journal of Real Estate Finance and Economics 14(3), 333-340.

Thanos, S., J. Dubé, and D. Legros (2016). Putting time into space: the temporal coherence of spatial applications in the housing market. Regional Science and Urban Economics 58, 78 - 88.

Wilhelmsson, M. (2002). Spatial models in real estate economics. Housing, Theory and Society 19(2), 92-101. 


\section{SUPPLEMENTARY: A GRID BASED APPROACH TO THE BOSTON DATASET}

Figure 5: A Grid Based Approach to the Harrison and Rubinfeld (1978) Boston Dataset

(a) Posterior Model Probabilities

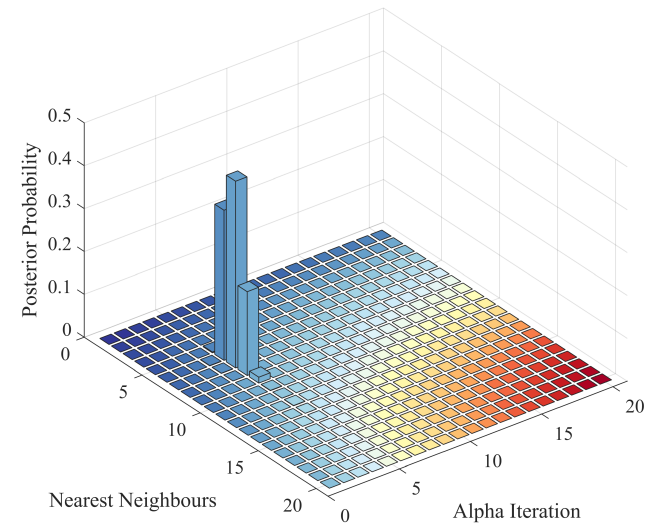

(c) Log Likelihoods

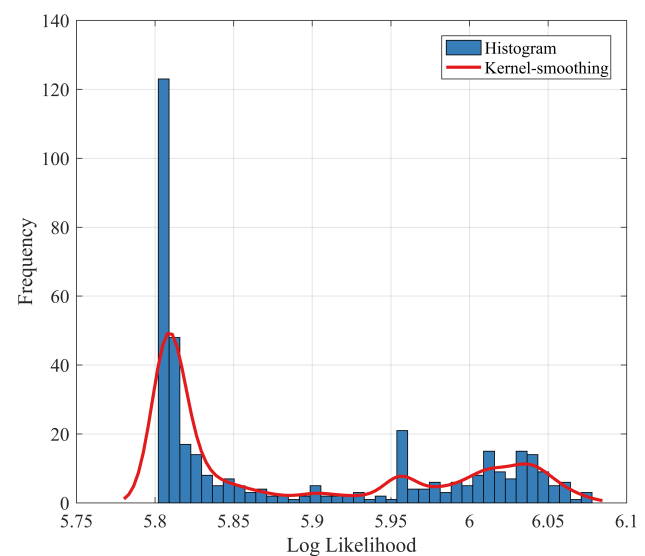

(b) Direct Effects: Crime)

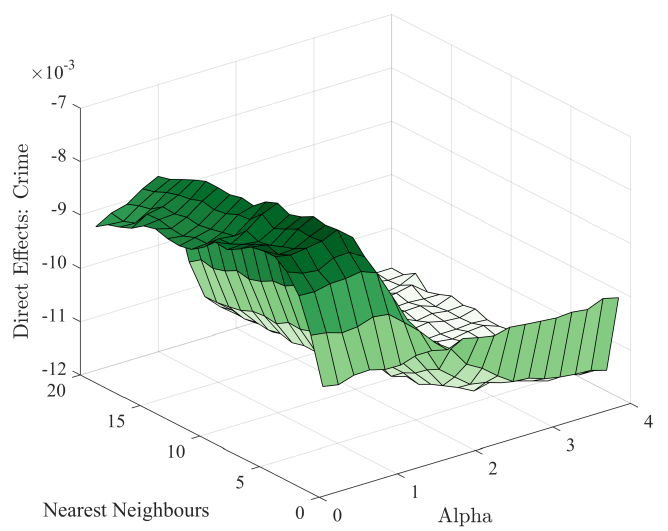

(d) $\hat{\lambda}$ Estimates

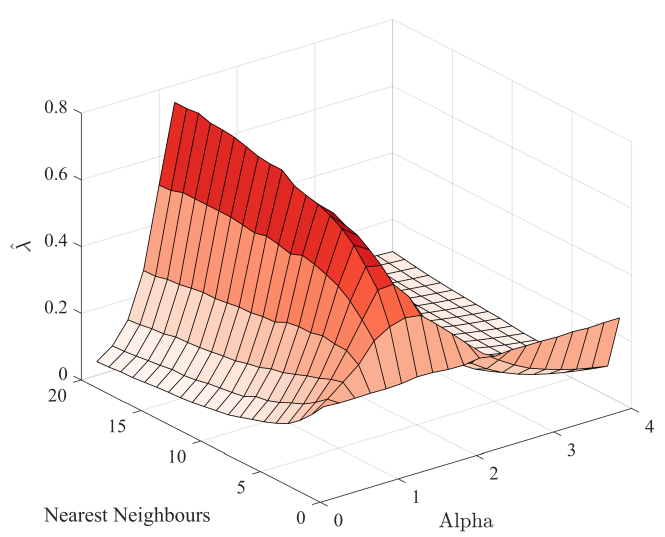

We emphasize the viability of this approach by estimating a simple set of models across a grid comprised of nearest neighbor and alpha variation using a popular, open-source dataset. This can be seen as a development of Section 3.3 of LeSage and Pace (2014) and Kostov (2010), both based on the Pace and Gilley (1997) amendments which augmented the infamous Harrison and Rubinfeld (1978) data with spatial co-ordinates. We follow the ten-variable specification of LeSage and Pace (2014), and transform the variables accordingly. We then follow their weighting scheme $W_{i, j}=\frac{1}{d(i, j)_{m}^{\alpha}}$, where $d(i, j)_{m}$ denotes the distance between the $m$ nearest neighboring observations between $j$ and $i$ and $\alpha$ is the decay parameter. We vary the grid between 1-20 nearest neighbors and across 20 alpha intervals between 0.4-4 to show that despite a strong posterior preference for a small number of models, a high variance in the log-likelihoods and the $\lambda$ parameter, the direct effects (Crime) stay relatively constant between -0.0119 and -0.0079 . 